\title{
MACHEAMENTO DO ORIFÍCIO PILOTO: ANÁLISE MECÂNICA NA VÉRTEBRA DE CARNEIRO E NO MODELO DE OSSO ARTIFICIAL
}

\author{
TAPPING PILOT HOLE: MECHANICAL ANALYSIS OF SHEEP \\ VERTEBRA AND THE ARTIFICIAL BONE MODEL
}

Patrícia Silva', Rodrigo César Rosa', Antonio Carlos Shimano², Francisco José Albuquerque de Paula³ José Batista Volpon ${ }^{4}$, Helton Luiz Aparecido Defino ${ }^{4}$

\section{RESUMO}

Objetivo: Avaliar a influência do macheamento do orifício piloto associado às outras variáveis como o seu diâmetro em relação ao diâmetro interno do parafuso e o modo de preparo no torque de inserção, na resistência ao arrancamento dos parafusos utilizados para a fixação anterior da coluna cervical. Método: Vinte corpos de prova de poliuretana e 30 vértebras torácicas (T1-T5) foram testadas. Em cada corpo de prova foram realizados quatro orifícios, sendo dois com diâmetro de 2,0mm e dois com 2,5mm. Esses orifícios foram confeccionados com broca ou sonda, de acordo com o grupo experimental. O macheamento do orifício piloto era ou não realizado, dividindo desse modo o grupo experimental em subgrupos iguais. Foram formados oito grupos experimentais (quatro utilizando poliuretana e quatro utilizando vértebras de carneiro). Parafusos corticais com 3,5mm de diâmetro externo e $14 \mathrm{~mm}$ de comprimento foram inseridos nos orifícios piloto. O torque de inserção foi mensurado durante a implantação dos parafusos e, em seguida, foram realizados ensaios mecânicos de arrancamento em máquina universal de testes $\mathrm{Emic}^{\circledR}$, software Tesc 3.13, célula de carga de $1.000 \mathrm{~N}$, velocidade de aplicação de força de $0,2 \mathrm{~mm} / \mathrm{min}$, pré-carga de $5 \mathrm{~N}$ e tempo de acomodação de 10 segundos. A propriedade avaliada nos ensaios mecânicos foi a força máxima de arrancamento. Resultados e Conclusão: O macheamento do orifício piloto reduziu significativamente o torque de inserção e a força de arrancamento dos parafusos em todos os grupos experimentais.

Descritores - Coluna vertebral; Parafusos ósseos; Biomecânica; Torque; Dispositivos de fixação ortopédica

\section{ABSTRACT}

Objective: To determine the effect of pilot hole tapping, together with other variables such as pilot hole diameter, in relation to inner screw diameter and preparation mode, on the insertional torque and pullout strength of the screws used for anterior fixation of the cervical spine. Methods: Twenty polyurethane test bodies and 30 thoracic vertebrae (T1-T5) were tested. Four holes were drilled into each test body, two of them with a diameter of $2.0 \mathrm{~mm}$ and two with a diameter of $2.5 \mathrm{~mm}$. The holes were drilled with a bit or probe according to the experimental group. Pilot hole tapping was or was not done, dividing the experimental group into equal subgroups. Eight experimental groups were formed, four of which used polyurethane specimens and four used sheep vertebrae. Cortical screws $3.5 \mathrm{~mm}$ in outer diameter and $14 \mathrm{~mm}$ long were inserted into the pilot holes. Insertional torque was measured during screw implantation and mechanical assays were then performed using an Emic ${ }^{\circledR}$ universal testing machine. Tesc 3.13 software was used for data analysis. Load cells with a capacity of $1000 \mathrm{~N}$, were used and the rate of force application was $0.2 \mathrm{~mm} / \mathrm{min}$. The mechanical property evaluated was the maximum pullout force of the screws. Results and Conclusions: Pilot hole tapping significantly decreased insertional torque and pullout force in all experimental groups.

Keywords - Spine; Bone screws; Biomechanics; Torque; Orthopedic fixation devices

1 - Pós-graduando da Faculdade de Medicina da Universidade de São Paulo - USP - Ribeirão Preto (SP), Brasil.

2 - Professor Associado do Departamento de Biomecânica, Medicina e Reabilitação do Aparelho Locomotor da Faculdade de Medicina da Universidade de São Paulo - USP - Ribeirão Preto (SP), Brasil.

3 - Professor Associado do Departamento de Clínica Médica da Faculdade de Medicina da Universidade de São Paulo - USP - Ribeirão Preto (SP), Brasil.

4 - Professor Titular do Departamento de Biomecânica, Medicina e Reabilitação do Aparelho Locomotor da Faculdade de Medicina da Universidade de São Paulo - USP Ribeirão Preto (SP), Brasil.

Trabalho realizado no Laboratório de Bioengenharia da Faculdade de Medicina da Universidade de São Paulo - USP - Ribeirão Preto (SP), Brasil.

Correspondência: Helton L.A. Defino - Rua Dornélia de Souza Mosca, 235, Jardim Canadá - 14024-120 - Ribeirão Preto (SP), Brasil. E-mail: hladefin@fmrp.usp.br 


\section{INTRODUÇÃO}

A fixação da coluna cervical é utilizada para fornecer estabilidade mecânica ao segmento vertebral durante o processo de consolidação da artrodese ${ }^{(1)}$. A estabilidade da fixação cervical depende de vários fatores, como a densidade mineral óssea, torque de inserção e força de arrancamento do parafuso ${ }^{(2-6)}$.

O torque de inserção e a força de arrancamento dos parafusos podem ser influenciados pelo macheamento do orifício piloto, existindo relatos não concordantes na literatura com relação a esse tópico ${ }^{(7-10)}$. Os efeitos negativos do macheamento do orifício piloto na resistência ao arrancamento têm sido demonstrados, especialmente, em corpos de prova de baixa dureza e no osso trabecular ${ }^{(7,11)}$. Na coluna lombar, o macheamento do orifício piloto reduziu significativamente a resistência ao arrancamento dos parafusos pediculares ${ }^{(12,13)}$. Entretanto, Ronderos et al ${ }^{(9)}$ observaram que o macheamento do orifício piloto não aumentou a força axial de arrancamento quando os parafusos cervicais anteriores eram ancorados na cortical posterior do coro vertebral. Carmouche et al $^{(14)}$ observaram que o macheamento reduzia a resistência ao arrancamento dos parafusos pediculares fixados na coluna lombar humana e não alterava a força de arrancamento dos implantes na coluna torácica.

O objetivo do estudo foi avaliar a influência do macheamento do orifício piloto associado às outras variáveis como o seu diâmetro, em relação ao diâmetro interno do parafuso, e o modo de preparo do orifício piloto, no torque de inserção e na resistência ao arrancamento dos parafusos utilizados para a fixação anterior da coluna cervical.

\section{MÉTODO}

O estudo foi realizado em corpos de prova de poliuretana, modelo de osso artificial, e vértebras de carneiro da raça Santa Inês deslanados com peso médio de $38 \pm$ $5 \mathrm{~kg}$, idade de $12 \pm 3$ meses. Foram utilizados 20 corpos de prova de modelo de osso artificial com 40mm de comprimento, $40 \mathrm{~mm}$ de largura e $40 \mathrm{~mm}$ de altura com densidade de $0,32 \mathrm{~g} / \mathrm{cm}^{3}$ (Nacional Ltda.), e 30 vértebras do segmento T1-T5 de carneiro, com densidade de 0,6 $\pm 0,03 \mathrm{~g} / \mathrm{cm}^{2}$. A densidade das vértebras foi obtida pelo exame dual-energy radiograph absorptiometry (DEXA) e sistema $Q D R$ com software versão 11 - 2:5 (Hologic 4500 W, Waltham, MA, EUA).
Em cada corpo de prova eram realizados quatro orifícios, sendo dois com diâmetro de 2,0mm e dois com diâmetro de 2,5mm. Esses orifícios eram confeccionados com broca ou sonda, de acordo com o grupo experimental. Em cada grupo experimental, metade dos orifícios com o mesmo diâmetro eram macheados, utilizando-se macho de 3,5mm de diâmetro (Synthes ${ }^{\circledR}$ ). Na outra metade, os parafusos eram inseridos sem o macheamento prévio.

Parafuso cortical de $3,5 \mathrm{~mm}$ de diâmetro externo, $2,5 \mathrm{~mm}$ de diâmetro interno e $14 \mathrm{~mm}$ de comprimento (Synthes ${ }^{\circledR}$ ) foram inseridos no orifício piloto (Figura 1).

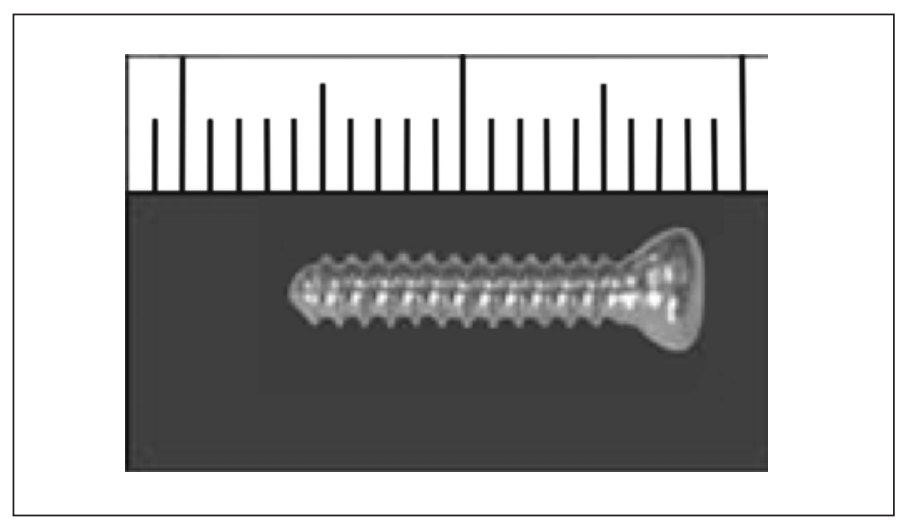

Figura 1 - Parafuso utilizado no estudo

Foram formados oito grupos experimentais (quatro utilizando modelo de osso artificial e quatro vértebras de carneiro). Grupos utilizando o modelo de osso artificial: I (orifício perfurado com broca de 2,0mm de diâmetro); II (orifício perfurado com broca de $2,5 \mathrm{~mm}$ de diâmetro); III (orifício perfurado com sonda de 2,0mm de diâmetro); e IV (orifício perfurado com sonda de 2,5mm de diâmetro). Grupos utilizando vértebra: $\mathrm{V}$ (orifício perfurado com broca de 2,0 mm de diâmetro); VI (orifício perfurado com broca de 2,5mm de diâmetro); VII (orifício perfurado com sonda de 2,0mm de diâmetro); e VIII (orifício perfurado com sonda de 2,5mm de diâmetro).

O torque de inserção dos implantes foi mensurado utilizando microtorquímetro digital da marca MK, modelo TI-500/MKMT-1, 1 N.m com capacidade de resolução de 0.001 N.m e foi utilizado o software Graphic III para a análise dos dados.

Os ensaios mecânicos foram realizados utilizando máquina universal de ensaio Emic ${ }^{\circledR}$, com célula de carga com capacidade de $1.000 \mathrm{~N}$, e os dados foram analisados por meio do software Tesc 3.13. 
Para a realização dos ensaios mecânicos de arrancamento, a cabeça do parafuso foi fixada à máquina de teste por meio de conectores que permitiam movimentos multidirecionais e a aplicação de carga axial de tração sem a aplicação de torque. Pré-carga de $5 \mathrm{~N}$ foi aplicada por um período de 10 segundos para acomodação do sistema e, então, carga axial de tração foi aplicada na constante de $0,2 \mathrm{~mm} / \mathrm{min}$ até o arrancamento do implante (Figura 2).

Dez ensaios mecânicos e 10 medidas do torque de inserção foram realizados para cada grupo experimental utilizando modelo de osso artificial (10 macheados e 10 não macheados). No total foram realizados 80 testes mecânicos e 80 medidas de torque de inserção. Para os grupos experimentais do corpo vertebral foram realizados

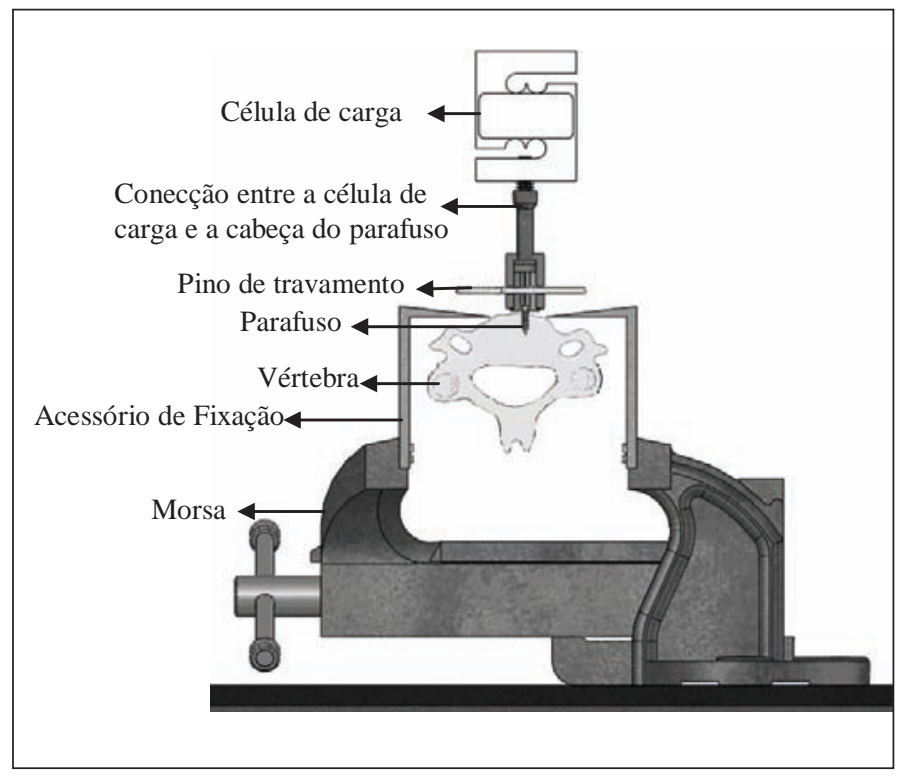

Figura 2 - Esquema dos acessórios utilizados nos ensaios mecânicos
15 ensaios mecânicos e 15 medidas de torque de inserção (15 macheados e 15 não macheados) sendo um total de 120 ensaios mecânicos e 120 mensurações de torque de inserção. A propriedade mecânica avaliada nos ensaios mecânicos foi a força máxima de arrancamento.

Os resultados foram submetidos ao teste de análise de variância multifatorial (Anova) usando o PROC GLM software SAS version 9 foi estabelecido nível de significância de $5 \%(\mathrm{p} \leq 0,05)$.

\section{RESULTADOS}

\section{Torque de inserção}

A média do torque de inserção dos parafusos implantados no modelo de osso artificial e no corpo vertebral está representada na Tabela 1 e Figura 3.

Tabela 1 - Valores médios e desvio padrão do torque de inserção dos parafusos implantados nos orifícios piloto macheados e não macheados no modelo de osso artificial e nos corpos vertebrais. O nível de significância estabelecido foi $p<0,05$

\begin{tabular}{|c|c|c|c|c|}
\hline \multirow[b]{2}{*}{ Material } & \multirow{2}{*}{$\begin{array}{c}\text { Grupos } \\
\text { experimentais }\end{array}$} & \multicolumn{2}{|c|}{ Torque de inserção } & \multirow[b]{2}{*}{$P$ valor } \\
\hline & & $\begin{array}{c}\text { Não macheado } \\
\text { (N.m) }\end{array}$ & $\begin{array}{l}\text { Macheado } \\
\text { (N.m) }\end{array}$ & \\
\hline \multirow{4}{*}{$\begin{array}{l}\text { Modelo de } \\
\text { osso artificial }\end{array}$} & I (Broca - 2,0mm) & $0,15 \pm 0,027$ & $0,03 \pm 0,006$ & $<0,001$ \\
\hline & II (Broca - 2,5mm) & $0,15 \pm 0,018$ & $0,03 \pm 0,008$ & $<0,001$ \\
\hline & III (Sonda - 2,0mm) & $0,16 \pm 0,017$ & $0,03 \pm 0,007$ & $<0,001$ \\
\hline & IV (Sonda - 2,5mm) & $0,17 \pm 0,028$ & $0,03 \pm 0,005$ & $<0,001$ \\
\hline \multirow{4}{*}{$\begin{array}{l}\text { Corpo } \\
\text { vertebral }\end{array}$} & V(Broca - 2,0mm) & $0,22 \pm 0,053$ & $0,07 \pm 0,048$ & $<0,001$ \\
\hline & VI (Broca - 2,5mm) & $0,18 \pm 0,051$ & $0,07 \pm 0,035$ & $<0,001$ \\
\hline & VII (Sonda - 2,0mm) & $0,25 \pm 0,061$ & $0,06 \pm 0,027$ & $<0,001$ \\
\hline & VIII (Sonda - 2,5mm) & $0,21 \pm 0,038$ & $0,05 \pm 0,016$ & $<0,001$ \\
\hline
\end{tabular}
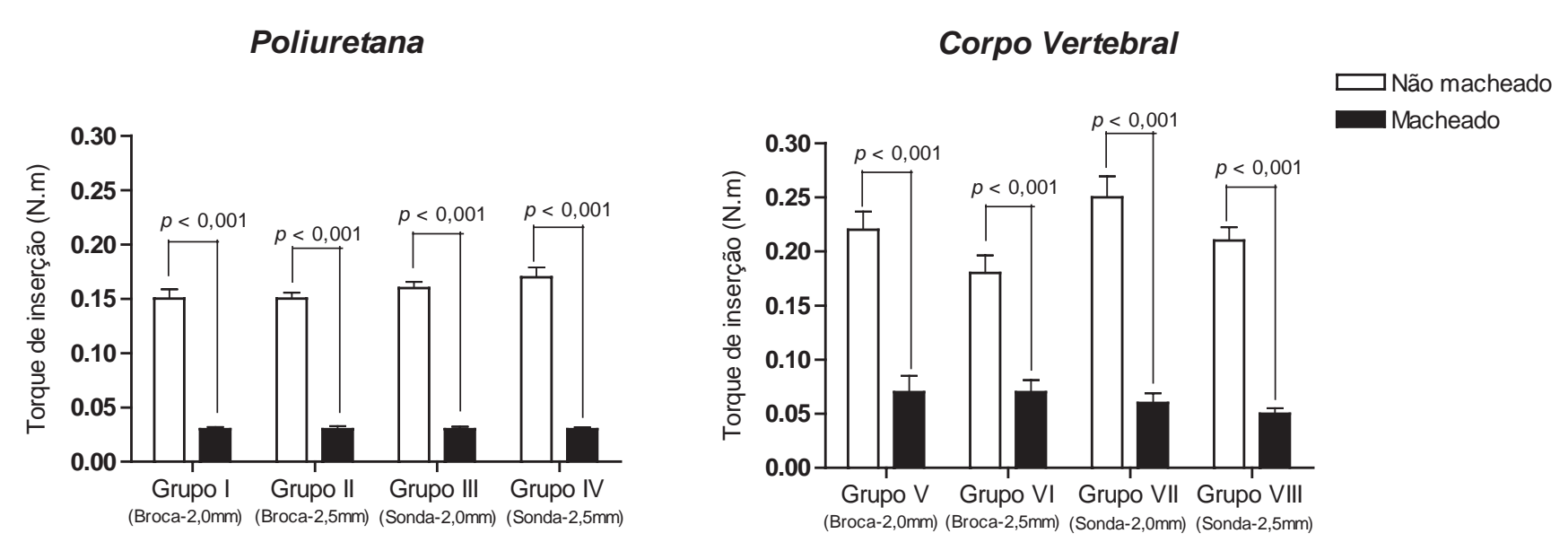

Figura 3 - Valores médios do torque de inserção dos parafusos implantados nos orifícios piloto macheado e não macheado no modelo de osso artificial e nos corpos vertebrais. O nível de significância estabelecido foi $p<0,05$ 
Foi observada redução significativa dos valores de torque de inserção dos implantes fixados nos orifícios piloto previamente macheados quando comparados com os não macheados em todos os grupos experimentais.

\section{Força de arrancamento}

A média da força de arrancamento dos parafusos implantados no modelo de osso artificial e no corpo vertebral está representada na Tabela 2 e Figura 4.

Foi observada redução significativa dos valores de força máxima de arrancamento dos implantes fixados nos orifícios piloto previamente macheados quando comparados com os não macheados em todos os grupos experimentais.

Tabela 2 - Valores médios e desvio padrão da força de arrancamento dos parafusos implantados nos orifícios piloto macheado e não macheado no modelo de osso artificial e nos corpos vertebrais. $O$ nível de significância estabelecido foi $p<0,05$

\begin{tabular}{|c|c|c|c|c|}
\hline \multirow[b]{2}{*}{ Material } & \multirow[b]{2}{*}{ Grupo experimental } & \multicolumn{2}{|c|}{ Força de arrancamento } & \multirow[b]{2}{*}{$P$ valor } \\
\hline & & $\begin{array}{c}\text { Não macheado } \\
\text { (N) }\end{array}$ & $\begin{array}{l}\text { Macheado } \\
\text { (N) }\end{array}$ & \\
\hline \multirow{4}{*}{$\begin{array}{l}\text { Modelo } \\
\text { de osso } \\
\text { artificial }\end{array}$} & I (Broca - 2,0mm) & $411,85 \pm 14,69$ & $369,58 \pm 11,98$ & $<0,001$ \\
\hline & II (Broca - 2,5mm) & $406,04 \pm 12,95$ & $356,40 \pm 7,96$ & $<0,001$ \\
\hline & III (Sonda - 2,0mm) & $451,48 \pm 18,67$ & $384,94 \pm 15,72$ & $<0,001$ \\
\hline & IV (Sonda - 2,5mm) & $412,29 \pm 33,33$ & $339,85 \pm 44,92$ & $<0,001$ \\
\hline \multirow{4}{*}{$\begin{array}{c}\text { Corpo } \\
\text { vertebral }\end{array}$} & $\mathrm{V}($ Broca $-2,0 \mathrm{~mm})$ & $374,43 \pm 83,10$ & $277,98 \pm 72,33$ & $=0,001$ \\
\hline & VI (Broca - 2,5mm) & $379,71 \pm 76,52$ & $259,30 \pm 42,29$ & $<0,001$ \\
\hline & VII (Sonda - 2,0mm) & $515,08 \pm 101,23$ & $338,07 \pm 77,61$ & $<0,001$ \\
\hline & VIII (Sonda - 2,5mm) & $372,55 \pm 98,36$ & $254,68 \pm 52,93$ & $<0,001$ \\
\hline
\end{tabular}

\section{DISCUSSÃO}

O parafuso cortical de 3,5mm utilizado nesse estudo foi o tipo de parafuso inicialmente empregado para as fixações da coluna cervical ${ }^{(14)}$. Essa modalidade de parafuso desenvolvido ainda é utilizada nas fixações posteriores da coluna cervical e, em menor escala, nas fixações anteriores, pois outros parafusos com desenhos e diâmetros mais adaptados ao osso esponjoso do corpo vertebral foram desenvolvidos ${ }^{(2)}$. Os mais recentes parafusos desenvolvidos não necessitam do macheamento (automacheantes) e/ou da perfuração (autoperfurantes) do orifício piloto reduzindo assim o trauma adicional ao paciente como também o tempo operatório ${ }^{(3,8)}$.

Os machos de rosca foram projetados para cortar o tecido ósseo reproduzindo exatamente o perfil do passo de rosca dos parafusos correspondentes. O macheamento do orifício piloto altera a composição interna do osso, produzindo fraturas da matriz do tecido ósseo esponjoso, favorecendo a formação de espaços nulos e reduzindo os componentes ósseos da interface osso-implante, o que dificultaria a sua ancoragem ${ }^{(7)}$. Outros estudos relatam que, apesar do macheamento do orifício piloto remover material ósseo, este processo não reduziria a força de arrancamento quando aplicados em osso do tipo cortical, ao contrário, facilitaria sua fixação ${ }^{(9,15)}$. Entretanto em ossos menos densos ou osteoporóticos foi observada redução de até $30 \%$ da força máxima de arrancamento ${ }^{(15)}$.

Os resultados obtidos no presente estudo mostraram que o macheamento do orifício piloto reduziu estatisticamente o torque de inserção e a força de arrancamento dos implantes em todos os grupos experimentais indepen-

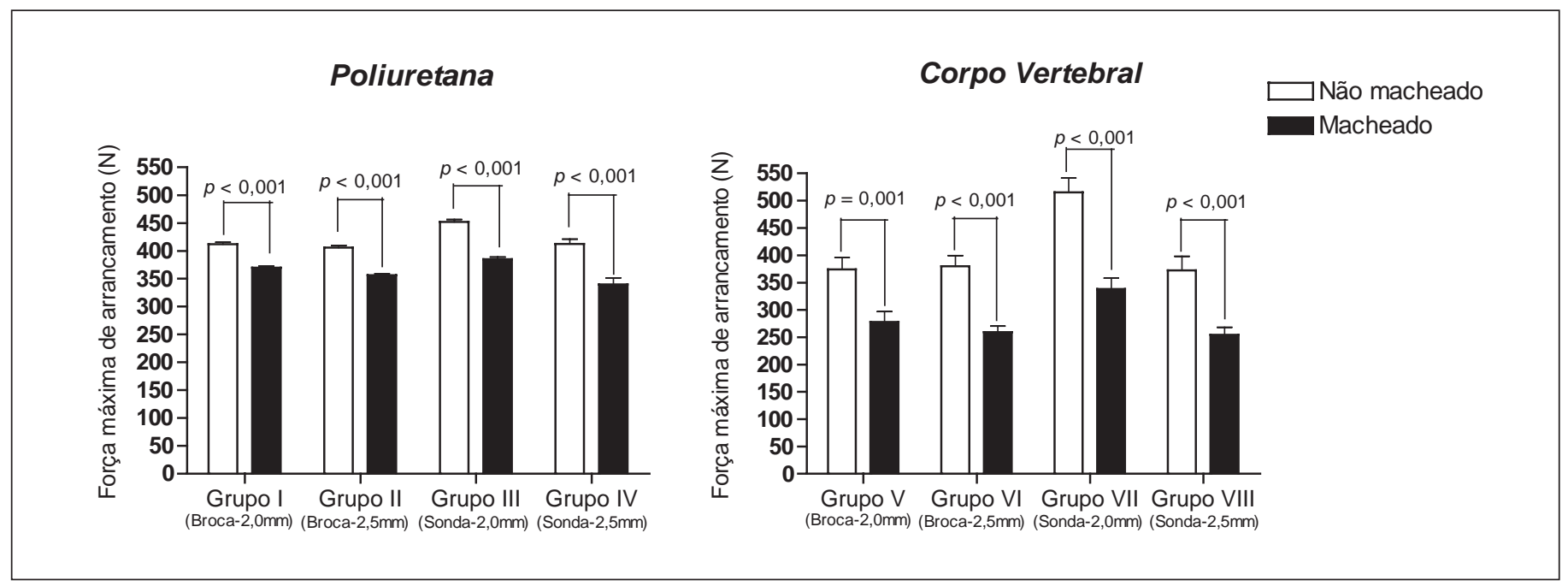

Figura 4 - Valores médios da força de arrancamento dos parafusos implantados nos orifícios piloto macheado e não macheado no modelo de osso artificial e nos corpos vertebrais. O nível de significância estabelecido foi $p<0,05$ 
dentemente do modo de preparo do orifício piloto e do diâmetro de perfuração em relação ao diâmetro interno do parafuso. A impactação do tecido ósseo adjacente ao implante promovida pelo uso da sonda ou perfuração do orifício piloto com diâmetro menor que o diâmetro interno do parafuso ${ }^{(7)}$ não foi suficiente para prevenir os efeitos negativos do macheamento. Contudo, o não macheamento do orifício piloto, além de diminuir o tempo cirúrgico, está associado a melhor ancoragem dos implantes.

As desvantagens do macheamento do orifício piloto relacionado à força de arrancamento dos parafusos têm sido bem demonstradas, especialmente em materiais macios ou em ossos esponjosos ${ }^{(7,9,11)}$. Os valores densitométricos de normalidade da coluna cervical estão bem reportados na literatura entre 0,304 e $0,343 \mathrm{~g} / \mathrm{cm}^{3(2)}$. As vértebras utilizadas no presente estudo, assim como os corpos de prova de modelo de osso artificial, possuíam densidade mineral óssea dentro dos limites de normalidade, com ausência de osteoporose ${ }^{(2)}$; contudo, os resultados obtidos mostraram redução no torque de inserção e na força de arrancamento dos implantes quando o orifício piloto era previamente macheado, independente dos parâmetros densitométricos dentro do limite de normalidade. Entretanto, Ronderos et al ${ }^{(9)}$, utilizando em seu estudo parafusos corticais para fixação anterior da coluna cervical, observaram que o macheamento não debilita e nem aumenta a força de arrancamento quando os mesmos são fixados bicorticalmente. Na coluna torácica não foi observada redução significativa na resistência ao arrancamento com várias técnicas de macheamento ${ }^{(9)}$.

O torque de inserção é o momento angular de força requerido para que o parafuso avance a rosca no interior do material de fixação ${ }^{(3)}$. O macheamento reduziu a força exigida durante o torque de inserção para cortar

\section{REFERÊNCIAS}

1. Dvorak MF, Pitzen T, Zhu Q, Gordon JD, Fisher CG, Oxland TR. Anterior cervical plate fixation: a biomechanical study to evaluate the effects of plate design, endplate preparation, and bone mineral density. Spine (Phila Pa 1976). 2005;30(3):294-301.

2. Conrad BP, Cordista AG, Horodyski M, Rechtine GR. Biomechanical evaluation of the pullout strength of cervical screws. J Spinal Disord Tech. 2005;18(6):506-10.

3. Hitchon PW, Brenton MD, Coppes JK, From AM, Torner JC. Factors affecting the pullout strength of self-drilling and self-tapping anterior cervical screws. Spine (Phila Pa 1976). 2003;28(1):9-13.

4. Pitzen T, Barbier D, Tintinger F, Steudel WI, Strowitzki M. Screw fixation to the posterior cortical shell does not influence peak torque and pullout in anterior cervical plating. Eur Spine J. 2002;11(5):494-9.

5. Ryken TC, Clausen JD, Traynelis VC, Goel VK. Biomechanical analysis of bone mineral density, insertion technique, screw torque, and holding strength of anterior cervical plate screws. J Neurosurg. 1995;83(2):325-9.

6. Ryken TC, Goel VK, Clausen JD, Traynelis VC. Assessment of unicortical and bicortical fixation in a quasistatic cadaveric model. Role of bone mineral density and screw torque. Spine (Phila Pa 1976). 1995;20(17):1861-7.

7. Oktenoğlu BT, Ferrara LA, Andalkar N, Ozer AF, Sarioğlu AC, Benzel EC. Effects of hole preparation on screw pullout resistance and insertional torque: a biomechanical study. J Neurosurg. 2001;94(1 Suppl):91-6. e preparar o local para a implantação do parafuso; esta redução foi observada na coluna torácica e lombar ${ }^{(8,9)}$.

O objetivo do presente experimento não foi simular exatamente as condições clínicas, mas fornecer medidas confiáveis referentes à ancoragem dos implantes. Os ensaios mecânicos realizados são do tipo estático, tendo a finalidade de avaliar a resistência mecânica ao arrancamento dos implantes por meio da aplicação de carga axial ao longo do seu eixo e permitir comparação simples e segura ${ }^{(9)}$. A falha na ancoragem dos implantes pode estar relacionada a vários fatores como a geometria do implante, densidade mineral óssea e a técnica de preparo do orifício piloto ${ }^{(7,9,14)}$.

A estabilidade do sistema de fixação é dependente da força de ancoragem dos implantes no osso, e falhas nesta fixação podem resultar na soltura do implante e, consequentemente, perda da estabilidade. O macheamento do orifício piloto para os parafusos corticais implantados na coluna vertebral é desvantajoso, porque além de aumentar o tempo cirúrgico diminui a resistência do implante ao arrancamento.

\section{CONCLUSÃO}

O macheamento do orifício piloto reduziu o torque de inserção e a força de arrancamento dos parafusos fixados nos diferentes corpos de prova (osso e modelo de osso artificial), independente do diâmetro de perfuração do orifício piloto (menor ou igual ao diâmetro interno do parafuso) e do seu modo de preparo (broca ou sonda).

\section{AGRADECIMENTOS}

Trabalho realizado com o apoio CAPES, CNPq e FAPESP.

8. Pfeiffer FM, Abernathie DL, Smith DE. A comparison of pullout strength for pedicle screws of different designs: a study using tapped and untapped pilot holes. Spine (Phila Pa 1976). 2006;31(23):E867-70.

9. Ronderos JF, Jacobowitz R, Sonntag VK, Crawford NR, Dickman CA. Comparative pull-out strength of tapped and untapped pilot holes for bicortical anterior cervical screws. Spine (Phila Pa 1976). 1997;22(2):167-70.

10. Zdeblick TA, Kunz DN, Cooke ME, McCabe R. Pedicle screw pullout strength. Correlation with insertional torque. Spine (Phila Pa 1976). 1993;18(12):1673-6.

11. Hearn TC, Surowik JF, Schatzker J. Effects of tapping on the holding strength of cancellous bone screw. Vet Comp OrthopTraumatol. 1992;5(1):10-2.

12. Kwok AW, Finkelstein JA, Woodside T, Hearn TC, Hu RW. Insertional torque and pull-out strengths of conical and cylindrical pedicle screws in cadaveric bone. Spine (Phila Pa 1976). 1996;21(21):2429-34.

13. Pfeiffer M, Gilbertson LG, Goel VK, Griss P, Keller JC, Ryken TC, et al. Effect of specimen fixation method on pullout tests of pedicle screws. Spine (Phila Pa 1976). 1996;21(9):1037-44.

14. Carmouche JJ, Molinari RW, Gerlinger T, Devine J, Patience T. Effects of pilot hole preparation technique on pedicle screw fixation in different regions of the osteoporotic thoracic and lumbar spine. J Neurosurg Spine. 2005;3(5):364-70.

15. Orozco DR, Llovet TJ. Osteosínteses en las fracturas de raquis cervical. Rev Ortop Traumatol. 1970;14:285-8. 